\title{
TAXA DE DISPAROS EM UMA REDE NEURAL MODELADA POR AUTÔMATO CELULAR
}

\author{
Rafael Ribaski Borges*, Kelly Cristiane Iarosz ${ }^{\dagger}$, Antonio Marcos Batista ${ }^{\ddagger}$, Ricardo \\ Luiz Viana ${ }^{\S}$, Sergio Roberto Lopes ${ }^{\ddagger}$, Iberê Luiz Caldas $₫$ \\ *Pós-Graduação em Ciências, Universidade Estadual de Ponta Grossa, 84030-900 \\ Ponta Grossa, Paraná, Brasil \\ ${ }^{\dagger}$ Pós-doutoranda CNPq - Brasil, University of Aberdeen Institute for Complex Systems and \\ Mathematical Biology (ICSMB) \\ ¥Departamento de Matemática e Estatística, Universidade Estadual de Ponta Grossa, 84030-900 \\ Ponta Grossa, Paraná, Brasil \\ ${ }^{\S}$ Departamento de Física, Universidade Federal do Paraná, 81531-990, Curitiba, Paraná, Brasil \\ ฯ Instituto de Física, Universidade de São Paulo, Caixa Postal 66316, 05315-970, São Paulo, SP, Brasil \\ Emails: rafael.ribaski@gmail.com, kiarosz@gmail.com, antoniomarcosbatista@gmail.com, \\ viana@fisica.ufpr.br, sergio.roberto.lopes@gmail.com, ibere@if.usp.br
}

\begin{abstract}
We study the firing rate in a model of cellular automaton for a neural network with electrical and chemical synapses. We proposed a model in which local connections in the network represent electrical synapses and the nonlocal simulate the chemical synapses. The non-local connections or shortcuts were inserted according to the model of complex network small world with a probability specified connection, also being possible to add a time delay in the simulations. The time delay is the time required to update the state variable of a neuron affected. The mechanism provides chemical synapses to conduct self-sustained neuronal firing, which can be periodic or not, depending on the insertion of the time delay. Considering only the electrical synapses, the dynamics of neuronal firing rapidly decays to zero.
\end{abstract}

Keywords- Neurodynamics, Neural Networks, cellular automaton.

\begin{abstract}
Resumo- Estudamos a taxa de disparos em um modelo de autômato celular para uma rede neural com sinapses elétricas e químicas. Foi proposto um modelo em que as conexões locais na rede representam as sinapses elétricas e, as não locais simulam as sinapses químicas. As conexões não locais ou atalhos foram inseridos de acordo com o modelo de rede complexa mundo pequeno com uma probabilidade de conexão especificada, também sendo possível a adição de um tempo de atraso nas simulações. O tempo de atraso representa o intervalo de tempo necessário para atualizar a variável de estado de um neurônio afetado. O mecanismo com sinapses químicas fornece um comportamento auto-sustentável de disparos neuronais, que pode ser periódico ou não, dependendo da inserção do tempo de atraso. Considerando somente as sinapses elétricas, a dinâmica de disparos decai rapidamente a zero.
\end{abstract}

Keywords— Neurodinâmica, Redes neurais, Autômato celular.

\section{Introdução}

O cérebro humano é uma rede complexa que contém dezenas de bilhões de neurônios e cada um deles é conectado com dezenas de milhares de outros neurônios(Gerstner and Kistler, 2002). Estas células possuem habilidade de propagar sinais e transmitir informações por meio de uma sequência disparos em vários padrões temporais (Dayan and Abbott, 2001). Essas comunicações neuronais, denominadas sinapses, são a base de processos complexos, como movimento, percepção sensorial, aprendizado, memória e emoções.

A estrutura das conexões do sistema nervoso de mamíferos apresenta um grande número de atalhos que ligam neurônios afastados, tornando assim, a distância média entre neurônios pequena. Contudo, ainda existe um alto agrupamento, que caracteriza a propriedade mundo pequeno (Zamora-López et al., 2009), (ZamoraLópez et al., 2010) . Watts e Strogatz (Watts and
Strogatz, 1998) propuseram um modelo de rede complexa, obtida por meio da religação de uma pequena parte das conexões de uma rede regular, que simula as características observadas em uma rede mundo pequeno.

A dinâmica local de uma rede neural pode ser descrita por vários modelos que vão desde equações diferenciais, como nos modelos de Hodkgin-Huxley (Hodgkin and Huxley, 1952) e Hindmarsh-Rose (Hindmarsh and Rose, 1984), redes de mapas acoplados (Rulkov, 2001) (Rulkov, 2002), a autômatos celulares (Copelli et al., 2002).

Dos modelos citados, o autômato celular é mais simples, pois são caracterizados por espaço, tempo e variáveis de estado discretas. As variáveis de estado de um autômato celular assumem valores em um conjunto discreto para cada posição espacial e cada instante de tempo (Wolfram, 1983). Estas variáveis são atualizadas simultâneamente de acordo com as variáveis de seus vizinhos por meio de uma regra determinística ou estocás- 
tica. Copelli e colaboradores (Copelli et al., 2002) utilizaram este modelo para investigar a faixa dinâmica em uma rede neural com sinapses elétricas.

Neste trabalho estudamos a taxa de disparos neuronais por meio de um modelo de autômato celular considerando sinapses elétricas e químicas. A dinâmica local do autômato celular descreve as sinapses elétricas, enquanto que, as sinapses químicas são obtidas pela dinâmica não local, onde as conexões seguem o modelo mundo pequeno de Newman e Watts (Newman and Watts, 1999). Também adicionamos um tempo de atraso nas sinapses químicas, o que representa o intervalo de tempo necessário para atualizar a variável de estado de um neurônio afetado.

\section{Modelo de rede Neural}

\subsection{Dinâmica local: Sinapses elétricas}

Seja $x_{i}(t), i=1,2, \ldots$, a variável de estado que caracteriza cada um dos neurônios no tempo discreto $t=1,2,3, \ldots$. Se $x_{i}$ é igual a zero, dizemos que o neurônio correspondente está no estado de repouso ou inativo. O neurônio dispara somente se $x_{i}$ é igual a um e o estado refratário equivale a valores $2 \leq x_{i} \leq \mu$, onde $\mu$ é um número inteiro. Um neurônio no estado refratário não pode disparar, mesmo que haja condições favoráveis para isto.

O modelo de autômato celular que descreve estas características segue as regras seguintes (Copelli et al., 2002):

1. Se $x_{i}(t)=0$ então $x_{i}(t+1)=h_{i}(t)$,

2. Se $x_{i}(t) \neq 0$ então $x_{i}(t+1)=\left[x_{i}(t)+1\right]$ $\bmod \mu$.

Portanto, se existe um estímulo, $h_{i}=1 \mathrm{e} \mathrm{o}$ neurônio que está em repouso $x_{i}(t)=0$ dispara, ou seja, $x_{i}(t+1)=1$ e permanece inativo pelos próximos $\mu-2$ instantes de tempo. O estímulo externo $I_{i}(t)$ que influencia a célula $i$ no tempo $t$ é dado por:

$$
I_{i}(t)=\sum_{\mu} \delta\left(t, t_{\mu}^{(i)}\right)
$$

onde $\delta(a, b)$ é a delta de Kronecker. O intervalo de tempo $t_{\mu+1}^{(i)}-t_{\mu}^{(i)}$ é distribuído exponencialmente de acordo com a média de uma distribuição Poissoniana e $\mu$ é um número inteiro associado ao período refratário dos neurônios (Copelli and Kinouchi, 2004).

Para um autômato com $N$ células e considerando apenas sinapses elétricas e pertubações aleatórias, a variável $h_{i}$ é dada por (Copelli et al., 2002):

$$
h_{i}(t)=1-\left[1-\delta\left(I_{i}(t), 1\right)\right] \prod_{j= \pm 1}\left[1-\delta\left(x_{i+j}(t), 1\right)\right],
$$

A densidade de neurônios disparando em um instante de tempo é dada por:

$$
\lambda=\frac{1}{N} \sum_{i=1}^{N} \delta\left(x_{i}(t), 1\right) .
$$

\subsection{Dinâmica não local: Inclusão de sinapses químicas}

Foi analisado o modelo de autômato celular considerando atalhos nas conexões segundo o modelo mundo pequeno de Newman e Watts (Newman and Watts, 1999). As conexões locais simulam as sinapses elétricas e, os atalhos inseridos, a dinâmica não local das sinapses químicas. Neste caso não foi considerado estímulos ou pertubações externas. Portanto a variável $h_{i}$ será dada por (Iarosz et al., 2012):

$$
\begin{array}{r}
h_{i}(t)=1-\left[1-\delta\left(x_{i-1}(t), 1\right)\right]\left[1-\delta\left(x_{i+1}(t), 1\right)\right] \\
\times \prod_{j \in J}\left[1-a_{i j} \delta\left(x_{j}(t-\tau), 1\right)\right]
\end{array}
$$

onde $\tau$ é o tempo de atraso e $J$ é o conjunto de vizinhos não locais escolhidos de forma aleatória na rede.

O último produto na Eq.(4) contém o elemento $a_{i j}$ da matriz adjacência, que neste caso é assimétrica, pois as conexões não locais são direcionadas. Foram utilizadas condições de contorno livres nas simulações: Os nós $x_{1}$ e $x_{N}$ possuem apenas uma conexão local e nenhuma não local, ou $x_{0}(t)=x_{N}(t)=0$ para todos os valores de $\mathrm{t}$. Com uma condição inicial $x_{i}(0)$, o sistema evolui por meio de uma sequência de padrões, que pode ou não convergir para um estado absorvedor homogêneo $x_{1}(t)=\ldots=x_{N}(t)=0$, isto é, não existe mais nenhum disparo dos neurônios.

\section{Resultados e discussões}

Para todas as simulações foi utilizada uma rede com $N=100$ neurônios com condição inicial $x_{50}=1$, com todos os outros neurônios inativos $\left(x_{i}=0\right.$ para $\left.i \neq 50\right)$ com a variável $h_{i}$ dada pela Eq.(4). Inicialmente apresentamos o resultado considerando somente a rede com conexões locais ( $p=0$, ou seja, somente sinapses elétricas) e tempo de atraso $\tau=0 . \quad \mathrm{Na}$ Fig.1(a) existe uma sequência de disparos de neurônios e seus estados refratários, representados pelos pontos pretos e verdes, respectivamente. Neste caso, os disparos são resultantes somente da atividade de neurônios vizinhos no instante anterior e cessam 
devido as condições de contorno livres. $\mathrm{Na}$ Fig.1(b) é apresentada a densidade de disparos dada pela Eq.(3), sendo constante inicialmente e convergindo a zero quando os disparos cessam.
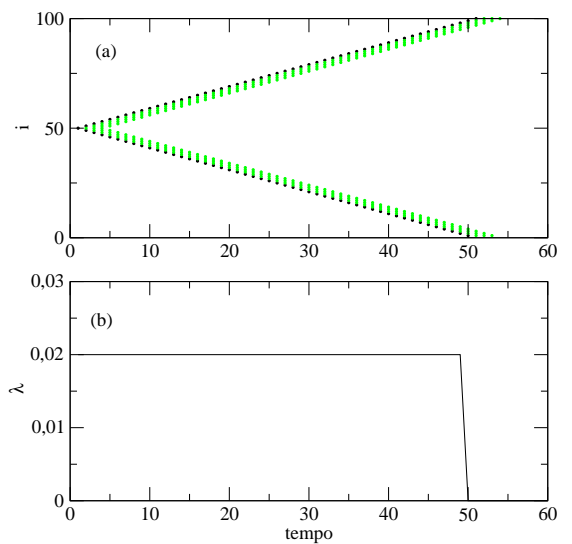

Figura 1: Evolução espaço-temporal da atividade neuronal para o modelo de autômato celular com $N=100$ neurônios e $\mu=5$, os pontos pretos correspondem aos neurônios disparando $\left(x_{i}=1\right)$, os verdes aos em estado refratário $\left(2 \leq x_{i} \leq \mu-1\right)$ e em branco aos que estão em repouso. (a) Considera-se $p=0$ e $\tau=0$ (somente sinapses elétricas). (b) Densidade de disparos para as condições de (a).

Considerando-se também as sinapses químicas, mas ainda com tempo de atraso $\tau=0$, foram introduzidas as conexões não locais com probabilidade $p=7.10^{-4}$. Na Fig.2(a) observa-se inicialmente um comportamento irregular de disparos, devido a influência das conexões não locais entre os neurônios. No entanto, uma sequência de disparos com comportamento periódico também pode ser observado pela densidade de disparos na Fig.2(b).

A adição de conexões não locais representando as ligações químicas asseguram que a atividade neuronal não cesse, configurando um mecanismo auto-sustentável de disparos.

Ainda considerando as sinapses químicas, foi inserido um tempo de atraso $\tau$ nas conexões não locais. Este artifício de adição de tempo de atraso frequentemente produz em um sistema não linear um comportamento complexo. Na Fig. 3(a) foi considerado $\tau=10$ e ainda $p=7.10^{-4}$. Observase que agora o comportamento dos disparos torna-se menos regular quando comparado com as simulações com tempo de atraso $\tau=0$. Este fato pode ser visualizado na evolução temporal da densidade de disparos, que apresenta um comportamento aperiódico ( Fig. 3(b)).
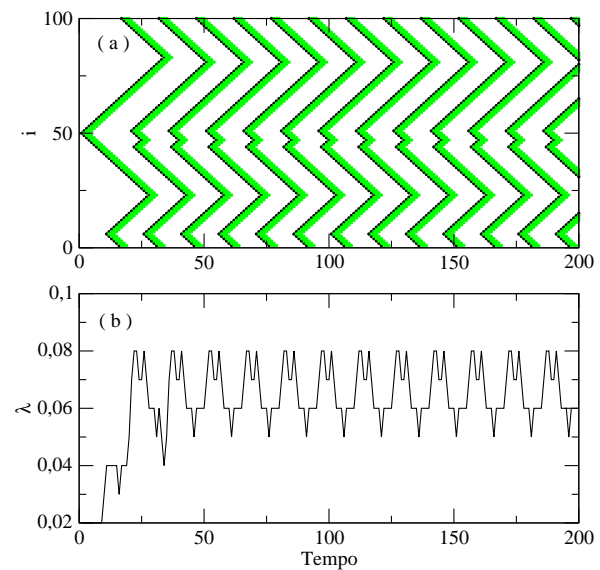

Figura 2: Evolução espaço-temporal da atividade neuronal para o modelo de autômato celular com $N=100$ neurônios e $\mu=5$ e a inclusão de sinapses químicas. (a) Considera-se $p=7.10^{-4}$ e $\tau=0$ (sem tempo de atraso). (b) Densidade de disparos para as condições de (a).
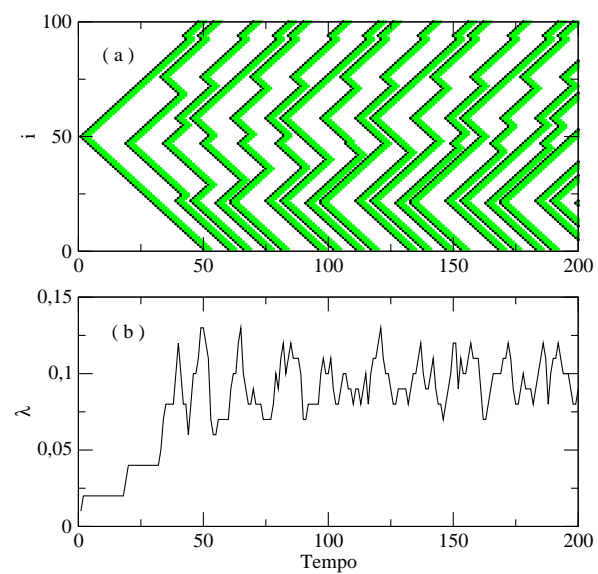

Figura 3: Evolução espaço-temporal da atividade neuronal para o modelo de autômato celular com $N=100$ neurônios e $\mu=5$ e a inclusão de sinapses químicas. (a) Considera-se $p=7 \cdot 10^{-4}$ e $\tau=10$. (b) Densidade de disparos para as condições de (a).

\section{Conclusões}

Neste trabalho apresentamos um modelo de autômato celular para simular disparos neuronais em uma rede neural com sinapses elétricas e químicas. As sinapses elétricas são modeladas pelas conexões locais, enquanto que, as sinapses químicas são inseridas pelas conexões não locais seguindo o modelo de rede complexa mundo pequeno de Newman-Watts, com uma probabilidade especificada. Para as conexões não locais pode ser ainda considerado um tempo de atraso, que é 
tempo necessário para a variável de estado do neurônio afetado seja atualizada. Considerando somente as sinapses elétricas, a taxa de disparos decai rapidamente a zero, devido as condições de contorno livres. No entanto, com a adição das sinapses químicas, o modelo exibe um comportamento auto-sustentável de disparos. Para tempo de atraso nulo, este comportamento dos disparos é periódico e considerando valores diferentes de zero, este comportamento torna-se aperiódico.

\section{Agradecimentos}

Este trabalho foi financiado pelas agências de fomento CAPES, CNPq e Fundação Araucária.

\section{Referências}

Copelli, M. and Kinouchi, O. (2004). Intensity coding in two-dimensional excitable neural networks, Physica A $\mathbf{3 4 9}$.

Copelli, M., Roque, A. C., Oliveira, R. F. and Kinouchi, O. (2002). Physics of psychophysics: Stevens and weber-fechner laws are transfer functions of excitable media, Physical Review E 65.

Dayan, P. and Abbott, L. F. (2001). Theoretical Neuroscience: Computational and Mathematical Modeling of Neural Systems, MIT Press.

Gerstner, W. and Kistler, W. M. (2002). Spiking Neuron Models Single Neurons, Populations, Plasticity, Cambridge University Press. DOI: 10.1017/CBO9780511815706

Hindmarsh, J. and Rose, R. M. (1984). A model of neuronal bursting using three coupled first order differential equations, Proc. Royal Soc. London B 221. DOI: 10.1098/rspb.1984.0024

Hodgkin, A. and Huxley, A. (1952). A quantitative description of membrane current and its application to conduction and excitation in nerve, J. Physiology 117.

Iarosz, K. C., Batista, A. M., Viana, R. L., Lopes, S. R., Caldas, I. L. and Penna, T. J. P. (2012). The influence of connectivity on the firing rate in a neuronal network with electrical and chemical synapses, Physica A 391.

Newman, M. E. J. and Watts, D. J. (1999). Scaling and percolation in the small-world network model, Physical Review E 60.

Rulkov, N. F. (2001). Regularization of synchronized chaotic bursts, Physical Review Letters 86.
Rulkov, N. F. (2002). Modeling of spikingbursting neural behavior using twodimensional map, Physical Review $E$ 65.

Watts, D. J. and Strogatz, S. H. (1998). Collective dynamics of 'small-world' networks, Nature 393.

Wolfram, S. (1983). Statistical mechanics of celular automata, Reviews of Modern Physics 55.

Zamora-López, G., Zhou, C. and Kurths, J. (2009). Graph analysis of cortical networks reveals complex anatomical communication substrate, Chaos 19.

Zamora-López, G., Zhou, C. and Kurths, J. (2010). Cortical hubs form a module for multisensory integration on top of the hierarchy of cortical networks, Frontiers in Neuroinformatics 4. 